\title{
https://doi.org/10.5585/exactaep.v19n1.13806
}

Check for updates

\section{LOS EFECTOS DE LA DEUDA Y LA RESTRICCIÓN FINANCIERA EN EL COMPORTAMIENTO DE LA INVERSIÓN DE LAS EMPRESAS BRASILEÑAS NO FINANCIERAS COTIZADAS EN B3}

\author{
THE EFFECTS OF DEBT AND FINANCIAL DISTRESS ON THE INVESTMENT BEHAVIOR OF \\ BRAZILIAN NON-FINANCIAL COMPANIES LISTED ON B3
}

Duterval Jesuka ${ }^{1}$

Vinicius Silva Pereira²

Antonio Sergio Torres Penedo 3

\author{
${ }^{1}$ Mestre em Administração - Universidade Federal de \\ Uberlândia - UFU, Faculdade de Gestão e Negócios - \\ FAGEN \\ adm.duterval@gmail.com \\ ${ }^{2}$ Doutor em Administração - Universidade Federal de \\ Uberlândia/UFU, Faculdade de Gestão e Negócios/ \\ FAGEN \\ viniciuss56@gmail.com \\ ${ }^{3}$ Doutor em Engenharia de Produção - Universidade \\ Federal de Uberlândia - UFU, Faculdade de Gestão e \\ Negócios - FAGEN \\ drpenedo@gmail.com
}

Recebido em: 03 maio 2019

Aprovado em: 09 out. 2019

Cite como - American Psychological Association (APA)

Jesuka, D., Pereira, V. S., \& Penedo, A. S. T. (2021, jan./mar.). Los efectos de la deuda y la restricción financiera en el comportamiento de la inversión de las empresas brasileñas no financieras cotizadas en B3. Exacta, 19(1), 130-149. https://doi.org/10.5585/exactaep.v19n1.13806.
Resumen: Este estudio investigó el impacto de las restricciones financieras sobre la relación entre el comportamiento de inversión y las decisiones de endeudamiento de las empresas brasileñas cotizadas B3. Se utilizó una regresión lineal con datos de panel en una muestra de 536 empresas que cotizaron en el período de 2010 a 2017. El tamaño de la empresa, la deuda a largo y corto plazo y la estructura de la propiedad se consideraron como variables de restricción financiera. Se encontró que, en Brasil las grandes empresas y privadas, poseen mayor proporción de activos, tienen menores restricciones para endeudarse, y aun así, deciden sobre sus inversiones basándose en la oportunidad de crecimiento y en sus capacidades de generar flujo de caja. Mientras tanto, las empresas pequeñas, con una pequeña deuda bancaria a largo plazo y las estatales encuentran más restricciones financieras, en consecuencia, sólo invierten a partir de flujos de caja generados.

Palabras clave: Estructura de Capital. Comportamiento de Inversión. Restricción Financiera.

Abstract: This study investigated the impact of financial restrictions on the relationship between investment behavior and debt decisions of Brazilian companies listed on B3. The authors performed a panel data regression in a sample of 536 listed companies from 2010 to 2017. The Company size, short and long-term bank debt and the ownership structure were considered financial constraints metrics. Empirical evidences show that in Brazil, large and private companies have a higher proportion of assets, have fewer restrictions on borrowing, and even so, they decide on their investments based on their growth opportunity and their ability to generate cash flow. Meanwhile, small companies with small long-term bank debt and state-owned firms face more financial constraints; consequently, they only invest based on the generated cash flows.

Keywords: Capital Structure. Investment Behavior. Financial Distress. 


\section{Introducción}

En las últimas décadas, las discusiones sobre la estructura de capital, financiación de la deuda y sus consecuencias se convirtieron en el centro de atención en el mundo académico, que busca comprender cómo las decisiones de deuda corporativa pueden influir en las compañías financieras. Miller y Modigliani (1958) señalan que no hay ninguna relevancia en la relación entre la financiación de la deuda y la inversión de las empresas cuando se trata de un mercado perfecto. En este contexto, Myers (1977) analiza el problema de la falta de inversión y demostró que las empresas con altas expectativas de crecimiento que tienen deudas muy arriesgado, no invierten en proyectos con un valor actual neto potencialmente positivo, para evitar el conflicto de intereses entre acreedores y accionistas.

Al mismo tiempo, Jensen y Meckling (1976), el análisis de la relación entre el costo de la agencia y la estructura de capital, señalaron que el flujo de caja libre de influir en las decisiones de inversión de las empresas con bajo crecimiento. Los enfoques de Miller y Modigiliani (1958) y Myers (1977) sirvieron como punto de partida para muchos autores investigan la relación entre la estructura de capital, deuda y decisiones de inversión empresarial (Haris \& Raviv, 1991; Aivazan et al., 2005a; González, 2007; Ahn et al., 2006; Alcokck et al., 2011; Dang, 2011; Khaw \& Lee, 2016; Phan, 2018).

Phan (2018) examinó el efecto de la deuda corporativa, las restricciones financieras sobre el comportamiento de inversión 435 empresas vietnamitas que figuran en la Bolsa de Vietstock entre 2010 y 2016. Además de los factores intrínsecos relacionados, el autor trató de comprender la forma de financiación de la deuda afecta a la elección inversión de las empresas en Vietnam. Después de esto, la lógica, la obra de Phan (2018) sirvió de base para investigar esta relación en Brasil.

Muchos autores han estudiado los aspectos relacionados con la estructura de capital y deuda de las empresas en el país, sin embargo, no hay necesidad de que haya una investigación actualizada del impacto de la deuda y las limitaciones financieras en el comportamiento de la inversión empresarial, teniendo en cuenta sus implicaciones para las empresas del Estado y privada. Este trabajo tiene como objetivo contribuir a la literatura para ampliar la discusión sobre este tema.

La teoría del comportamiento de la inversión desarrollada por Jorgensen y Siebert (2017) se refiere a las formas en que las empresas gestionan sus inversiones para aumentar el valor de mercado. Hay estudios empíricos que han estudiado el comportamiento de la inversión empresarial en diferentes países, siguiendo el movimiento de los gastos de capital y los activos fijos netos cada año (Elliott, 1973; Jorgenson \& Siebert, 2017). Los autores mostraron que las empresas de inversión, depende de sus características, su propiedad y sus perspectivas de crecimiento.

Basado en el trabajo Phan (2018), esta investigación pretende dar respuesta a la siguiente pregunta: ¿Cuál es la relación entre el comportamiento de la inversión y la cantidad / volumen y el vencimiento de la deuda de las empresas brasileñas no financieras cotizadas en B3 en la cara de las limitaciones financieras? Para responder a esta pregunta, este estudio tiene como objetivo analizar el 
impacto de las restricciones financieras sobre la relación entre el comportamiento de la inversión y las decisiones de endeudamiento de las empresas no financieras brasileñas que cotizan en la B3 en el período 2010-2017.

Par lograr este objetivo, se realizó una encuesta de las empresas que figuran en la B3 en el periodo de 2010 y 2017 en la base de datos Economática, en el que se excluyeron todas las empresas financieras y de seguros. Se eligió este periodo para esta investigación porque Brasil ha vivido momentos de inestabilidad política y económica que resultó en el aumento de la inflación y el interés fiscal tal como se señala en el informe del Banco Central (2017), hechos que podrían afectar a la deuda y la inversión empresarial. También se considera que por la Ley 11.941 / 2009 en la transparencia, las empresas comenzaron a publicar sus estados financieros a partir de 2010 como es requerido por la Comisión de Bolsa y Valores (CVM).

Este trabajo utiliza los conceptos principales de la estructura de capital para verificar el impacto de la deuda sobre el comportamiento de la inversión de las empresas, un aspecto que todavía tiene que ser explorada en la literatura, teniendo en cuenta el contexto actual de la crisis en Brasil. Otro factor importante de esta investigación es comprobar esta relación en las empresas públicas y privadas, a sabiendas de que la difícil situación financiera de las empresas públicas en el país que han sido privatizados.

Este trabajo sigue la siguiente estructura: inmediatamente después de esta sección de introducción, la segunda sección se presenta el fundamento teórico, la tercera sección se muestra la metodología utilizada en la cuarta sección se muestran y discutido los resultados de la investigación, las observaciones finales se hacen en la penúltima sección, y Este último contiene las referencias.

\section{Revisión de literatura}

En esta sección vamos a presentar la base teórica y empírica, así como la formulación de hipótesis que guían esta investigación.

\subsection{Financiación de la deuda y la inversión corporativa}

Contraer deuda para garantizar las inversiones futuras es un atributo para las empresas que tienen grandes perspectivas de crecimiento. Modigliani y Miller (1958) muestran que no existe una relación entre la deuda y el vencimiento de la deuda en un mercado perfecto, sin embargo, con la democratización del acceso al capital, las fuentes de financiación son diversificados y los factores externos generan imperfecciones, que es una consecuencia influye en el apalancamiento de las empresas. Myers (1977), crítica de las proposiciones de Modigliani y Miller (1958) demostró empíricamente que hay más bien una relación entre la deuda y las decisiones de inversión de las empresas. 
La relación entre el financiamiento de la deuda y la inversión corporativa ha sido objeto de estudio en varios países, mostrando que el apalancamiento de las compañías tiene un efecto significativo y negativo sobre la inversión (Albuquerque y Matias, 2016; Frank \& Shen, 2016; Mondosha \& Majoni, 2018; Nga, Dien, Linh \& Tuoi, 2019; Phan, 2018). Nga et al., (2019) utilizaron una muestra de 107 compañías que cotizan en la Bolsa de Ho Chi Minh (HOSE) de 2009 a 2014 para investigar la relación entre el apalancamiento y la inversión de las empresas vietnamitas, y encontraron un impacto positivo entre las dos variables y una relación negativa entre el vencimiento de la deuda y el crecimiento de la empresa.

Phan (2018) a su vez a prueba la hipótesis de la deuda afecta a las empresas de inversión con oportunidades de crecimiento bajas en 435 empresas que figuran en Vietnam entre 2010 y 2016. Los resultados no eran diferentes, se encontró que la ratio de apalancamiento tuvo un efecto negativo en la inversión de las empresas en Vietnam, esta es la mayor cantidad de deuda en la estructura de capital asociado con el bajo nivel de inversión.

En Brasil se observa que el mercado de capitales está menos desarrollado en comparación con otros países en este sentido, varios estudios demuestran preferencia por la deuda en la composición de la estructura de capital para financiar sus proyectos de crecimiento (Britto, Serrano \& Franco, 2018). En los últimos años, el país ha pasado por varias crisis económicas marcadas por las altas tasas de interés, que afectan a la elección de la deuda de muchas empresas, que como resultado habían entrado en proceso de des-apalancamiento. La relación entre la deuda y la inversión, también fue investigado por Albuquerque y Matias (2016), que analiza el impacto del apalancamiento de la inversión de las empresas públicas de Brasil, ambos se encuentran una relación negativa.

A partir de estos datos empíricos y proposiciones Myers (1977), se observa que la relación negativa entre la deuda y el vencimiento de la deuda en la inversión de las empresas, es debido al hecho de que el grado de apalancamiento puede llevar a conflictos de intereses entre los accionistas y los acreedores, lo que les impide invertir en proyectos que tienen un valor presente positivo, para evitar riesgos futuros con las deudas existentes. Por lo tanto, se espera que esta investigación que la cantidad de la deuda, así como la madurez tiene un efecto negativo en el comportamiento de la inversión empresarial, para comprobar esta hipótesis fórmula:

H1: La cantidad de la deuda y el vencimiento de la deuda tienen un efecto negativo sobre la inversión de empresas brasileñas.

\subsection{Las características de la empresa, la deuda y la inversión}

Uno de los problemas que pueden afectar a las decisiones de inversión de las empresas es el problema de agencia que se manifiesta por el conflicto de intereses entre accionistas y directivos. Esto requiere que los gerentes de invertir en proyectos futuros con el valor actual neto negativo de ampliar 
la escala de la empresa, sin embargo, el riesgo de liquidez puede influir en las decisiones de corto plazo, lo que limita la aplicación de esa política.

La recaudación de fondos a largo plazo puede estar influenciada por factores como la liquidez, el tamaño de la empresa, su relación con los bancos y su estructura de propiedad, además de estas características, se considera también el comportamiento de los inversores en el mercado de capitales. Varias investigaciones han investigado el impacto de las características de la empresa y del mercado en el comportamiento de la inversión corporativa. En este sentido, Frank y Shen (2016) analizaron la interacción con el flujo de caja, Jeon y Oh (2017) y Qi, Roth y Wald (2016) investigaron la relación de vencimiento de la deuda y la inversión, Lin y Chou (2015) investigaron la influencia de las normas institucionales banco, así como préstamo de créditos bancarios y comerciales.

\subsubsection{Tamaño: grandes y pequeñas empresas}

El tamaño de las empresas afecta a las condiciones de financiación de las empresas, así como el vencimiento de la deuda. Myers (1977) ha señalado que las grandes empresas tienen más oportunidades para financiar pequeñas en comparación. Phan (2018) destacó tres factores clave que son considerados por los prestamistas en la decisión de invertir sus fondos, a favor de las grandes empresas en relación con los menores. En primer lugar, las grandes empresas tienen más facilidades para negociar sus reclamos con los prestamistas que las pequeñas empresas. En segundo lugar, por tener una estructura administrativa sólida, las grandes empresas se enfrentan a un menor número de problemas de agencia y asimetría de la información, el riesgo de impago y los costos de transacción relativamente pequeña, por lo que los prestamistas prefieren prestar a esta categoría. Y por fin, las grandes empresas tienen más activos fijos que pueden usarse como garantía cuando se trata de su financiación a largo plazo con instituciones financieras.

Al igual que en otros países, en Brasil el tamaño influye en las posibilidades de financiación de las empresas (Almendra, Vasconcelos, Aragão y Cysne, 2017). Reis, Campos y Pasquini, (2017), analizaron el impacto de los determinantes de la estructura de capital en los ciclos de vida de las empresas, y se encontró una relación positiva entre el tamaño y el apalancamiento, lo que indica que esta variable está muy en cuenta en la concesión de préstamos a las empresas en el país. Almendra et al. (2017) analizaron la relación entre la estructura de capital y las inversiones en innovación de 120 empresas brasileñas que cotizan en BM\&FBovespa en el período de 2013 a 2015. Utilizando una regresión lineal múltiple, descubrieron que el tamaño influye positivamente en las decisiones de inversión de las empresas.

Estos trabajos y otros, han demostrado en el hecho de que el tamaño de las empresas es un factor importante en la decisión de la deuda pronto y de negocio a corto plazo, cuál es el análisis fundamental de sus relaciones en esta investigación. Por lo tanto, se infiere que el efecto de la deuda 
y la financiación de la deuda sobre el comportamiento de la inversión es diferente entre las empresas grandes y pequeñas, y para trabajar mejor esta hipótesis:

H2: La relación entre la deuda y las empresas de inversión es más sensible en las grandes empresas que en las pequeñas.

\subsubsection{Vencimiento de la deuda}

El préstamo a largo plazo es uno de los mecanismos de financiación que las empresas con un alto crecimiento potencial uso para recaudar fondos e invertir en sus proyectos. Cuando el acceso al mercado de capitales es limitado, recurrirá a los bancos es una de las alternativas que se adopte. Por lo tanto, la concesión de crédito corporativo a largo plazo se ve influida por factores macroeconómicos y las características de las empresas que restringen el acceso a la financiación bancaria (Phan, 2018; Silva, Bezerra \& Lima (2017).

En los países emergentes, los bancos se animan y dan préstamo de negocios para ampliar las actividades económicas generadoras de desarrollo, sin embargo, la fluctuación de los tipos de interés y el aumento de los diferenciales bancarios son elementos que pueden provocar dificultades financieras y el riesgo de incumplimiento en el largo plazo. Sleifer y Vishny (1997) analizan el incentivo para préstamo bancario afecta el comportamiento de la inversión de las empresas chinas entre 1999 y 2009, encontró que el problema de agencia es un factor importante en el control de las empresas por los acreedores.

En Brasil, el préstamo corporativo a largo plazo siempre ha sido una actividad exclusiva del Banco Nacional de Desarrollo Económico y Social - BNDES. Sin embargo, el rápido crecimiento del crédito bancario en el período comprendido entre 2004 y 2008 debido a cambios en la estructura del sector bancario en el país, favoreció la expansión de los préstamos a empresas de otros bancos privados, lo que reduce la participación de BNDES en la cantidad de $22 \%$ de crédito por $16 \%$ destacan Silva, Bezerra y Lima (2017).

La evolución del sistema de crédito bancario se expandió mecanismos de acceso al crédito de compañías, pero los factores macroeconómicos y las condiciones externas siguen influyendo en el comportamiento de los bancos en los préstamos corporativos en el largo plazo debido a la inestabilidad de los tipos de interés y el aumento excesivo de la propagación. informes del Banco Central (2009) y (2017) muestran la evolución de las tasas de interés que se acumularon un promedio entre el $12 \%$ y el $14,6 \%$ por año.

En la visión Prates y Freitas (2013), esto genera, por un lado, la incertidumbre en la economía que resulta en la reducción drástica en la concesión de préstamos bancarios a largo plazo. Para las empresas que mantienen deuda bancaria a largo plazo frente al aumento excesivo de intereses por sus inversiones, en relación a los niveles de deuda, en riesgo de incumplimiento y por lo tanto afecta a sus relaciones con los bancos. Teniendo en cuenta estas condiciones anteriores, se espera que el 
comportamiento de la inversión difiere entre las empresas que tienen la deuda a largo plazo y los que no tienen que comprobar este aspecto formularon la hipótesis siguiente:

H3: La relación entre la deuda y la inversión corporativa es más sensible en las empresas de la deuda en el largo plazo que los que no tienen deuda a largo plazo.

\subsubsection{Empresas estatales y empresas privadas}

En la teoría de la estructura de propiedad desarrollada por Jensen y Meckling (1977), se supone que las decisiones de los accionistas controladores pueden influir en las decisiones de inversión y deuda de las empresas. En este trabajo, investigamos la relación entre la financiación de la deuda y el comportamiento de la inversión de las empresas estatales y privadas.

El efecto de la estructura de la propiedad en el comportamiento de las empresas estatales, ha sido el objeto de estudio en varios países en los que se analizaron estas características, la comparación con las empresas privadas (Lizal \& Svejnar 2002; Lin, Malatesta, \& Xuan, 2013; Lin \& Chou, 2015; Phan, 2018). En Brasil, algunos estudios han abordado la estructura de capital de las empresas estatales y sus ventajas con respecto privada, que materializan la posición privilegiada que ocupa en el mundo empresarial (Mamede et al., 2017; Novaes, 1990 Rückert, 1981).

Las empresas estatales brasileños salieron de la década del 30 en el contexto posterior a la crisis mundial, un período en que el país comenzó el proceso de industrialización materializado por la intervención del Estado en la economía para producir bienes de capital. Después de años de transformación y consolidación, estas empresas públicas comenzaron a operar en todos los sectores productivos de bienes intermedios, tales como acero, petróleo, petroquímica, generación de energía, telecomunicaciones, ferrocarriles, minería. Con una presencia significativa en 1976 había aproximadamente 573 empresas de propiedad estatal, de los cuales 483 no financiero, mientras que en 1983 esta cifra se había elevado a 683 estado entre los cuales 571 no financiero (Novaes, 1990; Rückert, 1981).

Con una muestra de 104 empresas cotizadas en el BM\&FBOVESPA, Mamede et al., (2017) estudiaron la relación entre el endeudamiento de las empresas estatales y no estatales brasileñas en el período de 2008 a 2014. Los autores encentraran una relación negativa entre el tamaño y el nivel de endeudamiento de las empresas, además, se dio cuenta de que el endeudamiento de las empresas privadas es menor que el de las empresas estatales.

A pesar de que las empresas públicas se ocupan de los riesgos eminentemente políticos, su sólida estructura de capital y el valor de la equidad en la relación privada, asegurar un buen apoyo financiero para protegerlos contra el riesgo de quiebra. Estas características únicas permiten que las empresas estatales gozan de los privilegios y lograr un acceso fácil a la deuda a largo plazo para invertir en sus proyectos, a diferencia de las empresas privadas. En este sentido, como Phan (2018), 
tiene la intención de analizar el comportamiento de la inversión y de la deuda entre estos dos grupos de empresas a esta fórmula es la siguiente hipótesis:

H4: La relación entre la deuda y el comportamiento de la inversión corporativa es más sensible a las empresas privadas que las empresas estatales.

\section{Metodología}

En esta sección, se muestran los procedimientos metodológicos utilizados para esta investigación. Descriptivo y exploratorio, se utiliza el método cuantitativo y el panel de datos técnica de regresión lineal múltiple para llevar a cabo esta investigación.

\subsection{Muestra y datos}

Para lograr los objetivos de este trabajo fue considerado por primera vez la población de todas las empresas que cotizan en bolsa en Brasil que figuran en B3, procedentes de todos los sectores de la base de datos Economática en el período de 2010 a 2017 para observar el comportamiento de la inversión de éstos las empresas que consideran que el país entró en recesión después de la crisis política y económica. Entonces, como Dang (2011) y Phan (2018), fueron descartados muestra de todas las empresas financieras, debido a sus características particulares y el sector empresarial, lo que generó un total de 535 empresas con 4280 años-empresas observaciones para el análisis. Se utilizó la técnica de winsorizacíon para tratar la presencia de valores atípicos observó en las variables.

Para probar las hipótesis de esta investigación, la muestra se divide en tres sub-muestras en base a tres indicadores de restricción financieros, tales como: disponibilidad de préstamos a largo plazo, el tamaño y la estructura de propiedad de empresa. El primer sub-muestra examina la hipótesis 2, que se divide entre las empresas con deuda bancaria a largo plazo y los que no tienen deuda a largo plazo. Hipótesis 3 fue probado desde el tamaño de sub-muestra se separó usando la mediana del total de activos, es decir, empresas con activos totales por debajo de la mediana de la sub-muestra se consideran pequeñas, mientras que las situadas por encima se utilizan como las grandes empresas. Finalmente, en el caso de que el 4 sub-muestra de la estructura de propiedad se divide considerando un lado estado.

\subsection{Modelo empírico utilizado}

Para analizar el impacto de la deuda y financieros restricciones sobre el comportamiento de inversión de las empresas. En primer lugar, considerar el efecto de la relación entre el valor de mercado, el vencimiento de la deuda y el apalancamiento en el año anterior de la inversión para el año en curso. El modelo de regresión adoptado es el siguiente: 


$$
I N V_{i, t}=\beta o+\beta 1 D M A T_{i, t-1}+\beta 2 A P A L_{i, t-1}+\beta 3 Q_{i, t-1}+\beta 4 F C_{i, t}+\mu_{i}+\varepsilon_{i, t}
$$

En que:

$I N V_{i, t}:$ Inversión de la empresa i en el año t.

B0: modelo constante

$D M A T_{i, t-1}$ : Empresa vencimiento de la deuda i en el tiempo $t$

$\operatorname{APA} L_{i, t-1}$ : El apalancamiento financiero de la empresa i en el año t-1

$Q_{i, t-1}:$ Q de Tobin empresa i en el año t-1

$F C_{i, t}$ : El flujo de caja de la empresa i en el año t.

$\mu_{i}$ : Efecto individual de la empresa i en el tiempo-sin cambios

$\varepsilon_{i, t}:$ ¿Es las variaciones idiosincrásicas a los términos (el término de error)

A continuación, se considera el caso en el que las inversiones del año anterior pueden afectar las inversiones del año en curso y se añadió a la variable rezagada como variable explicativa. Por lo tanto, el modelo estadístico es el siguiente:

$$
I N V_{i, t}=\beta o+\beta 1 I N V_{i, t-1}+\beta 2 D M A T_{i, t-1}+\beta 3 A P A L_{, t-1}+\beta 4 Q_{i, t-1}+\beta 5 F C_{i, t}+\mu_{i}+\varepsilon_{i, t}
$$

Prueba de VIF se realizó (Varianza Inflación Factor), y el resultado 1,63 ser menos de 10, no mostró problemas de multicolinealidad. La aplicación de la prueba de Hausman y Chow agrupado con $\mathrm{F}<0,05$ probabilidad se encontró que el modelo de regresión más adecuado se de efectos fijos. $\mathrm{Y}$, finalmente, para verificar un problema heterodasticidad y autocorrelación se hicieron y la prueba de Wald y Woodridge rechazar la hipótesis nula (Ho), lo que indica que no hubo tales problemas entre las variables.

La Tabla 1 muestra las variables dependientes e independientes, como se usa en este trabajo de investigación por Barclay y Smith (1995), Kaplan y Zingales (1997), Aivazian (2005a y 2005b), Antoniou et al. (2006), Cai et al. (2008), Dang (2011), Alcock et al. (2012) y Phan (2018). 
Jesuka, D., Pereira, V. S., \& Penedo, A. S. T. (2020, out./dez.). Los efectos de la deuda y la restricción financiera en el comportamiento de la inversión de las empresas brasileñas no financieras cotizadas en B3

Tabla 1 - Variables y señales esperadas

\begin{tabular}{|c|c|c|c|c|c|}
\hline Variables & Abreviatura & $\begin{array}{l}\text { Método de } \\
\text { Medición }\end{array}$ & Fuente & Autores & $\begin{array}{c}\text { Signo } \\
\text { Esperado }\end{array}$ \\
\hline Inversíon & INV & $\begin{array}{c}\text { Gastos de capital } \\
\text { promedio / Activos } \\
\text { Fijos }\end{array}$ & Economática & $\begin{array}{l}\text { Aivazian, (2005a e } \\
\text { 2005b; Phan (2018), }\end{array}$ & \\
\hline $\begin{array}{l}\text { La madurez de } \\
\text { la deuda }\end{array}$ & DMAT & $\begin{array}{c}\text { A largo plazo } \\
\text { fundido deuda } \\
\text { Importe / Deuda } \\
\text { Total }\end{array}$ & Economática & $\begin{array}{c}\text { Cai et al. (2008), } \\
\text { Antoniou et al. (2006), } \\
\text { Alcock et al. (2012), }\end{array}$ & $(-)$ \\
\hline Apalancamiento & APAL & $\begin{array}{l}\text { La deuda total/ } \\
\text { Activo total }\end{array}$ & Economática & $\begin{array}{c}\text { Cai et al (2008), } \\
\text { Antoniou et al. (2006), } \\
\text { Alcock et al. (2012), } \\
\text { Barclay y Smith (1995) }\end{array}$ & $(-)$ \\
\hline $\begin{array}{l}\text { Crecimiento y } \\
\text { Oportunidades }\end{array}$ & Q & $\begin{array}{l}\text { El valor de } \\
\text { mercado de los } \\
\text { activos totales / } \\
\text { valor en libros de } \\
\text { los activos totales }\end{array}$ & Economática & $\begin{array}{c}\text { Kaplan y Zingales } \\
\text { (1997), Dang (2011), } \\
\text { Cai et al. (2008), } \\
\text { Antoniou et al. (2006), } \\
\text { Alcock et al. (2012) }\end{array}$ & $(+)$ \\
\hline Flujo de caja & FC & $\begin{array}{l}\text { Las ganancias } \\
\text { antes de intereses, } \\
\text { impuestos y } \\
\text { depreciación de } \\
\text { activos fijos / neto } \\
\text { al inicio del año }\end{array}$ & Economática & $\begin{array}{c}\text { Kaplan y Zingales, } 1997 \\
\text { Dang, (2011), Aivazian, } \\
\text { (2005a y 2005b) }\end{array}$ & $(+)$ \\
\hline
\end{tabular}

Fuente: Los autores.

\section{Análisis de los resultados}

En esta sección, se presentan las descripciones estadísticas y la matriz de correlación de las variables de estudio para el análisis previo del efecto de datos.

\subsection{El análisis descriptivo y matriz de correlación de las variables}

El análisis estadístico descriptivo se presenta en la Tabla 2. Como puede verse, la relación promedio de gastos de capital para los activos fijos al inicio del ejercicio (INV) de las empresas brasileñas es 0,73 con una desviación estándar de 0,95. Este resultado está por debajo de la media 0,24 encontrado por Phan (2018) a las empresas vietnamitas es, por tanto, cerca de las que se encuentran en los países desarrollados, 0,74 en los Estados Unidos Aivanzan (2005b) y 0,082 en el Reino Unido Dang (2011).

La ratio de deuda promedio a largo plazo de la deuda total (DMAT) es de 0,64, relativamente alta en comparación con los resultados encontrados por Phan (2018) 0,17 a empresas vietnamitas y cerca de los hallazgos en los países desarrollados como 0,66 para las empresas en los Estados Unidos Aivanzan (2005b), 0,74 en Australia Alcock (2010) y en Francia 0,59, 0,53 y 0,46 Alemania en el Reino Unido encontró por Antoniou et al (2006). La ratio de apalancamiento promedio (APAL) es 30.59 con 
una desviación estándar de 25.28. El promedio de la $Q$ de Tobin es de 0,67, lo que refleja las perspectivas de crecimiento del mercado.

Tabla 2 - Descripción de las variables estadísticas

\begin{tabular}{cccccc}
\hline & Nota. & Promedio & Desviacion Estandar & Min. & Max. \\
\hline INV & 2852 & 0.73 & 0.95 & 0.02 & 2.54 \\
DMAT & 3124 & 0.64 & 0.28 & 0 & 1 \\
APAL & 3767 & 30.59 & 25.28 & 0 & 98.68 \\
Q & 2103 & 0.67 & 0.57 & 0.08 & 1.90 \\
FC & 2817 & 2.34 & 3.30 & 0.07 & 8.49
\end{tabular}

Fuente: Los autores.

La Tabla 3 muestra la correlación entre las variables utilizadas en este estudio. Se puede observar que la correlación entre la madurez de la deuda (DMAT) y la proporción de la deuda (APAL) es 0,1568 positivo y significativo, lo que implica una relación positiva en la misma decisión de inversión corporativa. Sin embargo, se ve que las perspectivas de crecimiento de las empresas (Tobin $Q$ ) se correlaciona negativamente con sus decisiones de apalancamiento - 0,2964, lo que implica que el crecimiento del mercado influye negativamente en la deuda a largo plazo.

Tabla 3 - Matrix de Correlación

\begin{tabular}{cccccc}
\hline & INV & DMAT & APAL & Q & FC \\
\hline INV & 1.0000 & & & & \\
DMAT & $0,2261 *$ & 1.0000 & & & \\
APAL & $0,0435^{*}$ & $0,1568 *$ & 1.0000 & & \\
Q & $0,2051 *$ & $0,1179 *$ & $-0,2964 *$ & 1.0000 & \\
FC & $0,7038^{*}$ & $0,2143 *$ & $0,0536 *$ & $0,1942 *$ & 1.0000 \\
\hline
\end{tabular}

Fuente: Los Autores.

La tabla 4 muestra el resultado de la regresión al comportamiento brasileña empresas públicas de inversión en el período 2010 - 2017. Cabe señalar que las variables de influencia (APAL), retrasaron la inversión (INV), oportunidad de crecimiento ( $\mathrm{Q}$ Tobin) y flujo de caja (FC) presentaron los resultados de los efectos esperados en las empresas de inversión, mientras que la relación con el vencimiento de la deuda no fue significativa positiva. Sin embargo, a diferencia de las empresas vietnamitas Phan (2018), Aivazian (2005a, 2005b) y Dang (2011) Estados Unidos, no fue significativa el resultado de los coeficientes de inversión anteriores, la relación entre la deuda y el vencimiento de la deuda, lo que indica una débil influencia de estas variables sobre el comportamiento de la inversión de empresas brasileñas. 
Tabla 4 - Regresión de la muestra global

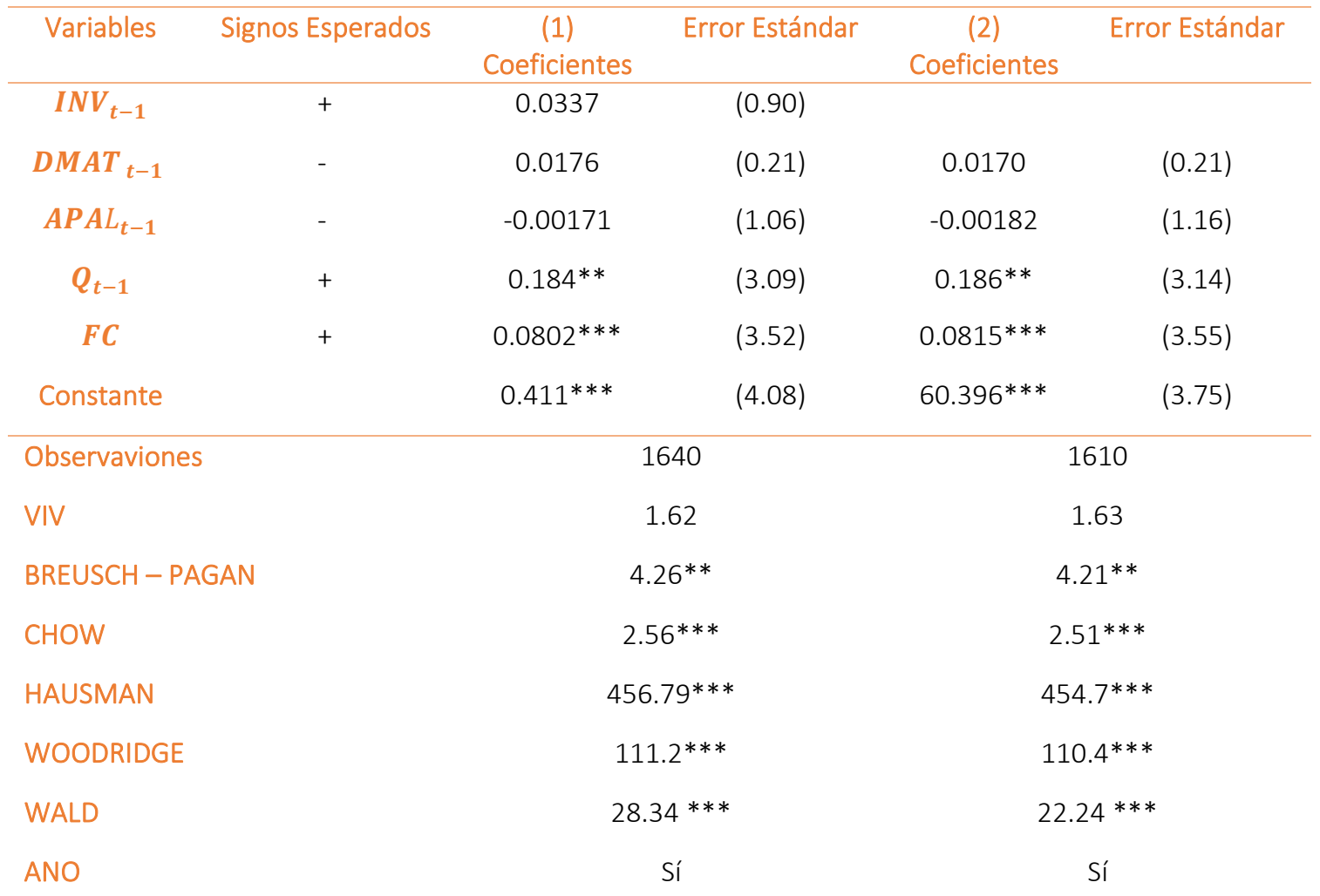

Nota: Nota: $* * *, * *$ y $*$ representan estadísticamente significativo al 1\%, 5\% y $10 \%$, respectivamente. Fuente: Los Autores.

El flujo de caja (FC) tuvo un efecto positivo y significativo en la decisión de inversión de las empresas, lo que indicaría un alto nivel de flujo de caja aumentaría más las empresas de inversión, que está de acuerdo con la investigación de Phan (2018). El resultado positivo y significativo de la Q de Tobin con la inversión muestra que la mayor de las expectativas de los inversores en acciones, la mayor inversión de la compañía. Estos resultados muestran que tanto el flujo de caja como oportunidades de crecimiento influye significativamente en el comportamiento de la inversión de las empresas públicas brasileñas.

El resultado de la regresión muestra que las empresas brasileñas en lugar de considerar el préstamo a largo plazo, además de dar prioridad a su capacidad de generar flujos de caja y oportunidades de crecimiento en el mercado para decidir sobre sus inversiones, como se ve en una relación positiva y significativa entre las variables asociada. Una explicación que, considere el hecho de que en el pasado Brasil ha sufrido varias crisis y la inestabilidad económica que dio lugar a la creciente influencia de la inflación en las tasas de interés. En consecuencia, las empresas se ven obligadas a optar por aumentar los fondos para sus proyectos de sus recursos internos y los mercados de capitales como una alternativa para escapar de las altas tasas de interés excesivas de la deuda a largo plazo. 


\subsection{Regresión para las empresas con y sin deuda a largo plazo}

Como se señaló, la disponibilidad de las influencias de los préstamos bancarios decisiones de inversión empresarial, como puede observarse en la Tabla 5, el resultado de la regresión muestra la relación entre el comportamiento de la inversión para las empresas con préstamos bancarios a largo plazo, así como las ellos no tienen este tipo de deuda en su estructura de capital. Cabe señalar que el flujo de caja se relaciona positiva y significativamente a los dos tipos de empresas, lo que indica que estas empresas deciden sobre sus futuras inversiones teniendo en cuenta la disponibilidad de recursos internos, este resultado es similar a Phan (2018) para las empresas vietnamitas. Al mismo tiempo,

A diferencia de Vietnam (Phan, 2018), la inversión en el año en curso de las empresas brasileñas sin deuda a largo plazo se ve influida negativamente y de manera significativa mediante el aprovechamiento y las inversiones realizadas en el anterior. Los coeficientes encontrados por sus variables retardadas indican que las empresas más apalancadas y son las mayores inversiones en el año anterior, menor será la inversión en el año en curso. Por otra parte la oportunidad de aumentar el crecimiento y el flujo de caja de inversión, porque las variables son resultados positivos y significativos. Para las empresas con deuda bancaria de las inversiones del año en curso siguen bajo la influencia positiva y significativamente por el crecimiento del año anterior y el flujo de caja generado en el ejercicio.

Tabla 5 - Las regresiones para empresas con/sin deuda a largo plazo

\begin{tabular}{|c|c|c|c|c|}
\hline \multirow{2}{*}{ Las Variables } & \multicolumn{2}{|c|}{ Empresa Sin Deuda a Largo Plazo } & \multicolumn{2}{|c|}{ Empresa Con Deuda a Largo Plazo } \\
\hline & Coeficientes & Error Estándar & Coeficientes & Error Estándar \\
\hline$I N V_{t-1}$ & $-0.173^{* * *}$ & (3.67) & 0.0326 & $(0.85)$ \\
\hline$D_{M A T_{t-1}}$ & 0.213 & $(1.64)$ & -0.0481 & $(0.52)$ \\
\hline$A P A L_{t-1}$ & $-0.00487 * * *$ & (2.81) & -0.000884 & $(0.51)$ \\
\hline$Q_{t-1}$ & $0.566^{* *}$ & (3.28) & $0.182^{* *}$ & $(3.07)$ \\
\hline$F C$ & $0.226 * * *$ & $(7.26)$ & $0.0812 * * *$ & (3.49) \\
\hline Constante & 0.361 & $(1.54)$ & $0.399 * * *$ & (3.59) \\
\hline Observaciones & \multicolumn{2}{|c|}{750} & \multicolumn{2}{|c|}{1535} \\
\hline VIF & \multicolumn{2}{|c|}{1.63} & \multicolumn{2}{|c|}{1.60} \\
\hline BREUSCH - PAGAN & \multicolumn{2}{|c|}{$4.26 * * *$} & \multicolumn{2}{|c|}{$4.18^{* * *}$} \\
\hline CHOW & \multicolumn{2}{|c|}{$2.56 * * *$} & \multicolumn{2}{|c|}{$2.16 * * *$} \\
\hline HAUSMAN & \multicolumn{2}{|c|}{$454.79 * * *$} & \multicolumn{2}{|c|}{$439.79 * * *$} \\
\hline WOODRIDGE & \multicolumn{2}{|c|}{$111,2 * * *$} & \multicolumn{2}{|c|}{$111.2 * * *$} \\
\hline WALD & \multicolumn{2}{|c|}{$2.234 * * *$} & \multicolumn{2}{|c|}{$2.34 * * *$} \\
\hline AÑO & \multicolumn{2}{|c|}{$454.79 * * *$} & \multicolumn{2}{|c|}{$452.9 * * *$} \\
\hline
\end{tabular}

Nota: $* * *, * *$ y representan estadísticamente significativo al $1 \%, 5 \%$ y $10 \%$, respectivamente. Fuente: Los Autores. 


\subsection{Regresión para grandes y pequeñas empresas}

Los resultados de la Tabla 6 muestran que el tamaño de la empresa asume una posición importante en la decisión de inversión de las empresas brasileñas. Se observa que el vencimiento de la deuda y el crecimiento, flujo de caja y la cantidad de deuda son insignificantes en la decisión de inversión de pequeñas empresas, sin embargo, las inversiones realizadas en el año anterior influencia negativa y significativamente las opciones de estas compañías. Estos resultados muestran la indiferencia de las pequeñas empresas brasileñas, así como Phan (2018) vietnamita, que son las limitaciones financieras para recaudar dinero para financiar sus futuros proyectos, debido al tamaño. Una explicación para esto, uno puede considerar el hecho de que estas empresas tienen menos activos tangibles e intangibles que pueden servir como garantía en las negociaciones del préstamo con los bancos.

Tabla 6 - Regresión para el tamaño de sub-muestra

\begin{tabular}{|c|c|c|c|c|}
\hline \multirow{2}{*}{ Las Variables } & \multicolumn{2}{|c|}{ Las Pequeñas Empresas } & \multicolumn{2}{|c|}{ Las Grandes Empresas } \\
\hline & Coeficientes & Error Estándar & Coeficientes & Error Estándar \\
\hline$I N V_{t-1}$ & $-0.190 *$ & $(2.5)$ & $0.0826 *$ & $(2.08)$ \\
\hline$D M A T_{t-1}$ & 0.133 & $(0.100)$ & -0.833 & $(0.77)$ \\
\hline$A P A L_{t-1}$ & -0.00127 & $(0.65)$ & -0.00313 & $(1.25)$ \\
\hline$Q_{t-1}$ & 0.0887 & $(1.04)$ & $0.201 * *$ & $(2.72)$ \\
\hline FC & -0.00667 & $(0.13)$ & $0.0812 * *$ & $(3.08)$ \\
\hline Constante & $0.208 *$ & (3.59) & $0.586 * * *$ & $(4.15)$ \\
\hline Observaciones & \multicolumn{2}{|c|}{495} & \multicolumn{2}{|c|}{1115} \\
\hline VIF & \multicolumn{2}{|c|}{1.63} & \multicolumn{2}{|c|}{1.59} \\
\hline BREUSCH-PAGAN & \multicolumn{2}{|c|}{$42.6^{* * *}$} & \multicolumn{2}{|c|}{$426.01 * * *$} \\
\hline CHOW & \multicolumn{2}{|c|}{$25.65 * * *$} & \multicolumn{2}{|c|}{$26.58 * * *$} \\
\hline HAUSMAN & \multicolumn{2}{|c|}{$447.9 * * *$} & \multicolumn{2}{|c|}{$454.79 * * *$} \\
\hline WOODRIDGE & \multicolumn{2}{|c|}{$101.25 * * *$} & \multicolumn{2}{|c|}{$111.2^{* * *}$} \\
\hline WALD & \multicolumn{2}{|c|}{$2.4 * * *$} & \multicolumn{2}{|c|}{$2.23 * * *$} \\
\hline AÑO & \multicolumn{2}{|c|}{ Sí } & \multicolumn{2}{|c|}{ Sí } \\
\hline
\end{tabular}

Nota: Nota: $* * *, * *$ y $*$ representan estadísticamente significativo al $1 \%, 5 \%$ y $10 \%$, respectivamente. Fuente: Los Autores.

Para las grandes empresas, la regresión mostró que el futuro comportamiento de la inversión está influenciado de manera positiva y significativa por la inversión anterior, la oportunidad para el crecimiento y el flujo de caja, mientras que el vencimiento de la deuda y la cantidad subieron simplemente no muestran valores significativos. El resultado tiene aspectos similares de empresas vietnamitas Phan (2018), mayor será la inversión y el crecimiento del año anterior, y el flujo de dinero 
en efectivo, mayor será la inversión para el año en curso. Cabe destacar que estas empresas tienen un mayor volumen de activos tangibles e intangibles, se enfrentan a una menor restricción para negociar sus préstamos con los prestamistas.

\subsection{Regresión para las empresas estatales y empresas privadas}

Para analizar el comportamiento de la inversión entre las empresas públicas y privadas, la muestra se divide en dos sub-muestras y eran dos regresiones, tal como se presenta en la Tabla 7. Sorprendentemente, se ha encontrado que las empresas de propiedad estatal sólo tenían el flujo de caja mostró una correlación positiva y significativa con el mismo comportamiento de la inversión. Por su parte, tanto el vencimiento de la deuda y el monto de la deuda y el crecimiento, así como las inversiones anteriores no tienen influencia significativa en las decisiones de inversión de las empresas estatales. En comparación con las empresas estatales vietnamitas, en el que Phan (2018) se ha encontrado evidencia que muestra que la inversión y el crecimiento rezagado.

Tabla 7 - La regresión a la estructura de propiedad

\begin{tabular}{|c|c|c|c|c|}
\hline \multirow{2}{*}{ Las variables } & \multicolumn{2}{|c|}{ Empresas Estatales } & \multicolumn{2}{|c|}{ Las Empresas Privadas } \\
\hline & coeficientes & Error Estándar & Coeficientes & Error Estándar \\
\hline$I N V_{t-1}$ & 0.129 & $(1.82)$ & 0.0228 & $(0.58)$ \\
\hline$D M A T_{t-1}$ & -0.596 & $(1.28)$ & 0.0368 & $(0.43)$ \\
\hline$A P A L_{t-1}$ & -0.0111 & $(1.53)$ & -0.00170 & $(1.04)$ \\
\hline$Q_{t-1}$ & -0.350 & $(1.77)$ & $0.187^{* *}$ & (3.07) \\
\hline$F C$ & $0.222 * *$ & (3.93) & $0.0702 *$ & (3.24) \\
\hline _cons. & 0.648 & $(1.08)$ & $0.412 * * *$ & (3.95) \\
\hline Observaciones & \multicolumn{2}{|c|}{124} & \multicolumn{2}{|c|}{1486} \\
\hline VIF & \multicolumn{2}{|c|}{1.63} & \multicolumn{2}{|c|}{1.54} \\
\hline BREUSCH-PAGAN & \multicolumn{2}{|c|}{$4.26 * * *$} & \multicolumn{2}{|c|}{$42.6 * * *$} \\
\hline CHOW & \multicolumn{2}{|c|}{$2.6 * * *$} & \multicolumn{2}{|c|}{$25.6^{* * *}$} \\
\hline HAUSMAN & \multicolumn{2}{|c|}{$44.71 * * *$} & \multicolumn{2}{|c|}{$45.79 * * *$} \\
\hline WOODRIDGE & \multicolumn{2}{|c|}{$111.2 * * *$} & \multicolumn{2}{|c|}{$110.8^{* * *}$} \\
\hline WALD & \multicolumn{2}{|c|}{$23.4 * * *$} & \multicolumn{2}{|c|}{$22.34 * * *$} \\
\hline AÑO & \multicolumn{2}{|c|}{ Sí } & \multicolumn{2}{|c|}{ Sí } \\
\hline
\end{tabular}

Nota: Nota: $* * *, * *$ y $*$ representan estadísticamente significativo al $1 \%, 5 \%$ y $10 \%$, respectivamente. Fuente: Los autores.

El resultado de la segunda regresión muestra que tanto el flujo de caja y el crecimiento son factores relevantes en las decisiones de inversión de las empresas privadas en Brasil, con coeficientes constantes positivas y significativas con los resultados de González (2007), mientras que la inversión, la 
madurez y la cantidad pasado de la deuda del año anterior son insignificantes en la elección de la inversión de estas empresas. Como Phan (2018) se esperaba que las empresas estatales brasileñas habían aumentado la disponibilidad de fondos para invertir en proyectos futuros en relación con las empresas privadas debido a la protección de impuestos y la disponibilidad de los activos tangibles e intangibles, sin embargo, se encontró que lo contrario. De hecho, las empresas estatales en Vietnam parecen ser más fuerte que el brasileño.

En este sentido, se puede considerar que la falta de confianza de los inversores y las instituciones financieras en el estado brasileño, que están influenciados por factores políticos, la corrupción y las amenazas a la posible privatización. Así que lo que tenemos como información es que, en Brasil las empresas siguen la jerarquía de las fuentes de financiación propuesto por Myers (1977) priorización de los recursos internos antes de embarcarse en el capital de deuda para decidir sobre sus inversiones.

\section{Consideraciones finales}

Este estudio tuvo como objetivo investigar los efectos de la deuda, así como las limitaciones financieras de las decisiones de inversión de las empresas brasileñas no financieras de capital de abeto que aparece en la B3 en varios aspectos, el análisis de la elección de la deuda se relaciona con el comportamiento de la inversión de las empresas. Basado en tres submuestras, se encontró que la relación teniendo en cuenta la disponibilidad de préstamos a largo plazo, el tamaño y la estructura de la propiedad, la realización, los resultados de la regresión presentaron pruebas para demostrar que la decisión de las empresas de inversión está influenciada por sus opciones de deuda, priorización de los recursos generados por el flujo de caja y oportunidades de crecimiento.

Los resultados muestran que las empresas tienden a reducir la cantidad de sus deudas para aumentar sus inversiones, sin embargo, y Dang (2011) para el Reino Unido y Phan (2018) a las empresas vietnamitas, vencimiento de la deuda no tiene ningún efecto significativo sobre el comportamiento de la inversión de las empresas. pruebas de que se encontraron empresas con deuda en el largo plazo, priorizar las oportunidades de crecimiento y los fondos generados por el flujo de caja para alimentar a sus inversiones actuales. Empresas sin deuda a largo plazo, aprovechar las oportunidades de crecimiento y el flujo de efectivo para invertir en sus proyectos.

Se encontró que el tamaño es un factor importante en el comportamiento de la inversión corporativa. Se ha observado que las grandes empresas con mayor proporción en activos, más capacidad de generar flujo de efectivo de las inversiones anteriores, no tienen en cuenta la cantidad de deuda o de vencimiento de la deuda en sus decisiones de inversión. Sin embargo, las pequeñas empresas que tienen más limitaciones financieras, no pueden generar recursos internos para financiar sus proyectos. Mediante el análisis de comportamiento de la inversión teniendo en cuenta la estructura de propiedad de las empresas, es que las empresas estatales invierten sólo en términos de 
su capacidad para generar flujo de caja, por su parte, tanto para el crecimiento privado y el flujo de caja son factores significativos en sus decisiones de inversión.

A partir de los resultados encontrados en este estudio, se puede decir que existe la necesidad de que el gobierno brasileño a adoptar políticas públicas que pueden garantizar un entorno económico estable y sostenible con bajas tasas de interés para reducir las incertidumbres que aumentan los préstamos bancarios a largo plazo para mejorar la disponibilidad de fondos para las empresas de apalancamiento, lo que favorece el empleo de generación y el crecimiento del país. Así como en los países en desarrollo, el gobierno debe fortalecer la estructura de las instituciones es fundamental para el desarrollo del sector de crédito en el país y el aumento de la competitividad en el escenario global (Muhanji \& Ojah, 2015; Cuadrado \& Japaridze, 2016; Gnangnon, 2013; Asiedu, 2003). Una buena regulación del sector bancario asociado a la deuda puede facilitar el apalancamiento de las empresas de González (2007).

Los bancos tienen que reducir las limitaciones financieras para facilitar el acceso a préstamos con tasas de interés más bajas para las pequeñas empresas que más sufren la falta de liquidez del mercado de capitales brasileño. Vale la pena mencionar la importancia que se debe dar las empresas estatales, que también ya sufren de limitaciones financieras, no debe ser utilizado como herramientas políticas, que pueden ser críticos para mejorar el acceso al capital para invertir en sus proyectos.

Este estudio tiene algunas limitaciones, entre las que podemos destacar el hecho de que no se ha considerado los efectos de las industrias, ya que pueden ser un factor relevante en el análisis del comportamiento de la inversión, la investigación futura podría considerar este aspecto. A medida que el comportamiento de la inversión se analizó sólo para las empresas públicas brasileñas que cotizan en la B3, otra investigación podría investigar esta relación mediante la comparación de las características que se encuentran en Brasil y otros países de América Latina, ya que tienen las mismas características del mercado.

\section{Referencias}

Ahn, S., Denis, J., \& Denis, K. (2006). Leverage and investment in diversified firms. Journal of financial Economics, 79(2), 317-337. https://doi.org/10.1016/j.jfineco. 2005.03.002

Aivazian, V., Ge, Y., \& Qiu, J. (2005). The impact of leverage on firm investment: Canadian evidence. Journal of corporate finance, 11(1-2), 277-291. https://doi.org/10.1016/S09291199(03)00062-2

Aivazian, V., Ge, Y., \& Qiu, J. (2005). Debt maturity structure and firm investment. Financial Management, 34(4), 107-119. https://doi.org/10.1111/j.1755-053X.2005.tb00120.x

Albuquerque, A., \& Matias, B. (2016). Impactos da maturidade, fonte e custo das dívidas no investimento de empresas brasileiras. In Anais do Congresso Brasileiro de Custos-ABC. Disponible en: https://anaiscbc.emnuvens.com.b r/anais/article/view/4183 
Alcock, J., Finn, F., \& Tan, K. (2012). The determinants of debt maturity in Australian firms. Accounting \& Finance, 52(2), 313-341.

Almendra, R. S., de Vasconcelos, A. C., Aragão, R. N., \& Cysne, I. A. (2017). Influência da estrutura de capital nos investimentos em inovação das indústrias listadas na BM\&FBOVESPA. Revista Eletrônica de Ciência Administrativa, 16(1), 40-61.

Alvarez-Cuadrado, F., \& Japaridze, I. (2017). Trickle-down consumption, financial deregulation, inequality, and indebtedness. Journal of Economic Behavior \& Organization, 134, 1-26.

https://doi.org/10.1016/j.jebo.2016.12.007

Antoniou, A., Guney, Y., \& Paudyal, K. (2006). The determinants of debt maturity structure: evidence from France, Germany and the UK. European Financial Management, 12(2), 161-194.

Asiedu, E. (2003). Debt relief and institutional reform: a focus on Heavily Indebted Poor Countries. The Quarterly Review of Economics and Finance, 43(4), 614-626. https://doi.org/10.1016/S10629769(03)00038-3

Barclay, J., \& Smith, W. (1995). The maturity structure of corporate debt. The Journal of Finance, 50(2), 609-631.. http://www.jstor.org/stable/2329421.

Britto, P. A. P., Serrano, A. L. M., \& Franco, V. R. (2018). Determinantes da estrutura de capital de empresas brasileiras de capital aberto em período de crise. Revista Ambiente Contábil, 10(2), 364383.

Cai, K., Fairchild, R., \& Guney, Y. (2008). Debt maturity structure of Chinese companies. Pacific-Basin Finance Journal, 16(3), 268-297. https://doi.org/10.1016/j .pacfin.2007 .06.001

Dang, A. (2011). Leverage, debt maturity and firm investment: An empirical analysis. Journal of Business Finance \& Accounting, 38(1-2), 225-258.

Elliott, W. (1973). Theories of corporate investment behavior revisited. The American economic review, 63(1), 195-207. http://www.jstor.org/stable/1803135

Frank, M. Z., \& Shen, T. (2016). Investment and the weighted average cost of capital. Journal of Financial Economics, 119(2), 300-315.

Gnangnon, K. (2013). Structural vulnerability and excessive public indebtedness in CFA Franc Zone countries. Economic Modelling, 35, 816-832. https://doi.org/10.1016/ j.econmod.2013.08.022

González, N. (2007). Banking regulation, institutional framework and capital structure: International evidence from industry data. The Quarterly Review of Economics and Finance, 47(4), 481-506..

Harris, M., \& Raviv, A. (1991). The theory of capital structure. the Journal of Finance, 46(1), 297-355.

Jensen, M., \& Meckling, H. (1976). Theory of the firm: Managerial behavior, agency costs and ownership structure. Journal of financial economics, 3(4), 305-360.

Jeon, H. J., \& Oh, H. M. (2017). Debt Maturity And Investment Efficiency Evidence From Korea. Journal of Applied Business Research (JABR), 33(6), 1081-1094.

Jorgenson, W., \& Siebert, D. (2017). Optimal capital accumulation and corporate investment behavior. Journal of Political Economy, 76(6), 1123-1151. http://www.jstor.org/stable/1830154 
Kaplan, N., \& Zingales, L. (1997). Do Investment-Cash Flow Sensitivities Provide Useful Measures of Financing Constraints? The Quarterly Journal of Economics, 112(1), 169-215. https://doi.org/10.2307/ 2951280

Khaw, H., \& Lee, J. (2016). Debt maturity, underinvestment problem and corporate value. Journal of Accounting \& Finance, 12. https://doi.org/10.21315/aamja f2016.12.s1.1

Lin, C., Ma, Y., Malatesta, P., \& Xuan, Y. (2013). Corporate ownership structure and the choice between bank debt and public debt. Journal of Financial Economics, 109(2), 517-534. https://doi.org/10.1016/j.jfineco.2013.03.006

Lin, T., \& Chou, J. (2015). Trade credit and bank loan: Evidence from Chinese firms. International Review of Economics \& Finance, 36, 17-29. https://doi.org/10. 1016/j.iref.2014.11.004

Lizal, L., \& Svejnar, J. (2002). Investment, credit rationing, and the soft budget constraint: Evidence from Czech panel data. Review of Economics and Statistics, 84(2), 353-370. https://doi.org/10.1162/003465302317411596

Prates, D., \& Freitas, M. (2013). Crédito bancário corporativo no Brasil: evolução recente e perspectivas. Brazilian Journal of Political Economy, 33(2), 322-340.

Mamede, S.P.N.; Nakamura, W.T.; Nakamura, E.A.M. \& Jones, G.D.C. (2017) Empresas Brasileiras Estatais e não Estatais uma Análise das Relações de Endividamento, Revista Evidenciação Contábil \& Finanças, 5(2), 4-22

Modigliani, F., \& Miller, H. (1958). The cost of capital, corporation finance and the theory of investment. The American Economic Review, 48(3), 261-297. http://www.jstor.org/stable /1809766

Mondosha, M., \& Majoni, A. (2018). The impact of leverage on investment decisions for South African firms with different growth opportunities. Journal of Economic and Financial Sciences, 11(1), 1-7.

Muhanji, S., \& Ojah, K. (2016). Governance infrastructure and indebtedness of African countries: Do regional blocs matter?. The North American Journal of Economics and Finance, 36, 123-153. https://doi.org/10.1016/j.najef.2015.12.004

Myers, S. (1977). Determinants of corporate borrowing. Journal of financial economics, 5(2), 147-175. https://doi.org/10.1016/0304-405X(77)90015-0

Nga, D. Q., Dien, P. M., Linh, N. T. C., \& Tuoi, N. T. H. (2019, January). Impact of Leverage on Firm Investment: Evidence from GMM Approach. In International Econometric Conference of Vietnam (pp. 282-295). Springer, Cham

Novaes, D. (1990). Rentabilidade e risco: empresas estatais versus empresas privadas. Revista brasileira de economia, 44(1), 53-84.

Phan, T. (2018). Corporate debt and investment with financial constraints: Vietnamese listed firms. Research in International Business and Finance, 46, 268-280.

https://doi.org/10.1016/j.ribaf.2018.03.004

Qi, Y., Roth, L., \& Wald, J. (2017). Creditor protection laws, debt financing, and corporate investment over the business cycle. Journal of International Business Studies, 48(4), 477-497. 
Reis, T., Campos, S., \& Pasquini, E. (2017). A influência dos determinantes da estrutura de capital conforme o estágio do ciclo de vida das empresas brasileiras. Revista de Gestão, Finanças e Contabilidade, 7(3), 127-142.

Rückert, J. (1980). Alguns aspectos das empresas estatais no Brasil. Ensaios FEE, 2(1), 75-93. https://revistas.fee.tche.br/index.php/ensaios/article/viewFile/87/417

Shleifer, A., \& Vishny, W. (1997). A survey of corporate governance. The journal of finance, 52(2), 737783. https://doi.org/10.1111/j.1540-6261.1997.tb04820.x

Silva, E., Bezerra, F., \& Lima, C. (2016). Determinantes de longo prazo do crédito no Brasil: liquidez versus capital bancário. Revista Brasileira de Finanças, 14(3), 375-402. 